\title{
New Solution to the Problem of a Crack in an Orthotropic Plate under Tension
}

\author{
V. V. Vasil'ev ${ }^{a, *}$, S. A. Lurie ${ }^{a, b}$, and V. A. Salov ${ }^{a}$ \\ ${ }^{a}$ Ishlinsky Institute for Problems in Mechanics of the Russian Academy of Sciences, Moscow, 119526 Russia \\ ${ }^{b}$ Institute of Applied Mechanics of Russian Academy of Sciences, 125040, Moscow, Russia \\ *e-mail:vvvas@dol.ru
}

Received March 17, 2021; revised March 22, 2021; accepted March 29, 2021

\begin{abstract}
A classical plane problem of the theory of elasticity about a crack in a stretched orthotropic elastic unbounded plane is considered, which leads to a singular solution for stresses in the vicinity of the crack edge. The relations of the generalized theory of elasticity, including a small scale parameter, are given. The equations of the generalized theory are of a higher order than the equations of the classical theory and allow eliminating the singularity of the classical solution. The scale parameter is determined experimentally. The results obtained determine the effect of the crack length on the bearing capacity of the plate and are compared with the experimental results for plates made of fiberglass and carbon fiber reinforced plastic.
\end{abstract}

Keywords: theory of elasticity, non-classical theory of elasticity, plane problem of a crack in an orthotropic plate

DOI: $10.3103 / \mathrm{S} 0025654421060200$

\section{INTRODUCTION - THE CLASSIC SOLUTION TO THE CRACK PROBLEM}

Let us consider an unrestricted orthotropic plate with a crack of length $2 c$ under conditions of uniaxial tension by stress $\sigma_{0}$ (Fig. 1). The stress-strain state of the plate is determined by the classical solution obtained by the method of complex potentials [1]. The stresses are determined by the equalities

$$
\begin{gathered}
\sigma_{x}=-p^{2} \operatorname{Re} \frac{A w_{1}}{\sqrt{w_{1}^{2}-c^{2}}}-q^{2} \operatorname{Re} \frac{B w_{2}}{\sqrt{w_{2}^{2}-c^{2}}}, \\
\sigma_{y}=\operatorname{Re} \frac{A w_{1}}{\sqrt{w_{1}^{2}-c^{2}}}+\operatorname{Re} \frac{B w_{2}}{\sqrt{w_{2}^{2}-c^{2}}} \\
\tau_{x y}=-p \operatorname{Im} \frac{A w_{1}}{\sqrt{w_{1}^{2}-c^{2}}}-q \operatorname{Im} \frac{B w_{2}}{\sqrt{w_{2}^{2}-c^{2}}} .
\end{gathered}
$$

Here, $A$ and $B$ are some constant coefficients, $w_{1}=x+i p y, w_{2}=x+i q y, p=1 / \sqrt{k_{1}}, q=1 / \sqrt{k_{2}}$ and $k_{1,2}$ are related to the roots of the characteristic equation corresponding to the generalized biharmonic equation of the plane problem, and are expressed in terms of the elastic constants of an orthotropic material as follows:

$$
k_{1,2}=E_{y}\left(\frac{1}{2 G_{x y}}-\frac{v_{y x}}{E_{x}}\right) \pm \sqrt{E_{y}^{2}\left(\frac{1}{2 G_{x y}}-\frac{v_{y x}}{E_{x}}\right)^{2}-\frac{E_{y}}{E_{x}}} \quad E_{x} v_{x y}=E_{y} v_{y x} .
$$

Let us take $y=0$ and consider the interval $-c<x<c$ corresponding to the boundaries of the crack (Fig. 1). It follows from equalities (1.1) and (1.2) that $\sigma_{x}=\sigma_{y}=0$ on this interval. Expression (1.3) allows us to conclude that the condition $\tau_{x y}=0$ at the crack boundary is satisfied if

$$
p A+q B=0 .
$$




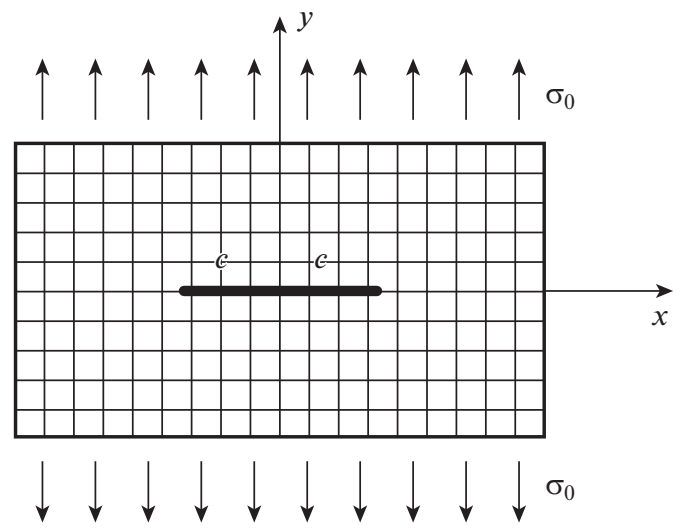

Fig. 1. Orthotropic plate with a crack.

At $|x| \rightarrow \infty$, the stress $\sigma_{y}$ should tend to $\sigma_{0}$ (Fig. 1). It can be shown that, in the limit, for any ray $y=k x$ hold the limit relations

$$
\lim _{x, y \rightarrow \infty} \frac{w_{1,2}}{\sqrt{w_{1,2}^{2}-c^{2}}}=1 .
$$

As a result, from the condition $\sigma_{y}(|x| \rightarrow \infty) \rightarrow \sigma_{0}$ we obtain $A+B=\sigma_{0}$. This condition, together with Eq. (1.4), gives

$$
A=-\frac{q \sigma_{0}}{p-q}, \quad B=\frac{p \sigma_{0}}{p-q} .
$$

However, equality (1.1) implies that for these values of the coefficients $\sigma_{x}$ also tends not to zero, but to $\sigma_{0}$ at $|x| \rightarrow \infty$. To eliminate this effect, the stress state of the plate, corresponding to Fig. 1, should be subjected to compression in the direction of the $x$ axis with a stress of $\sigma_{0}$ [1]. Finally, from equalities (1.1)(1.3) and (1.5) we obtain

$$
\begin{gathered}
\sigma_{x}=p q \sigma_{0}\left(\frac{p}{p-q} \operatorname{Re} \frac{w_{1}}{\sqrt{w_{1}^{2}-c^{2}}}+\frac{q}{p-q} \operatorname{Re} \frac{w_{2}}{\sqrt{w_{2}^{2}-c^{2}}}-1\right), \\
\sigma_{y}=-\frac{\sigma_{0}}{p-q} \operatorname{Re}\left(\frac{q w_{1}}{\sqrt{w_{1}^{2}-c^{2}}}-\frac{p w_{2}}{\sqrt{w_{2}^{2}-c^{2}}}\right), \\
\tau_{x y}=\frac{p q \sigma_{0}}{p-q} \operatorname{Im}\left(\frac{w_{1}}{\sqrt{w_{1}^{2}-c^{2}}}-\frac{w_{2}}{\sqrt{w_{2}^{2}-c^{2}}}\right) .
\end{gathered}
$$

On the real axis at $y=0$ and $x>c$, we have

$$
\begin{gathered}
\sigma_{x}=\sqrt{\frac{E_{x}}{E_{y}}} \sigma_{0}\left(\frac{x}{\sqrt{x^{2}-c^{2}}}-1\right), \\
\sigma_{y}=\frac{\sigma_{0} x}{\sqrt{x^{2}-c^{2}}} . \\
\tau_{x y}=0
\end{gathered}
$$

\section{EQUATIONS OF THE PLANE PROBLEM}

OF THE GENERALIZED THEORY OF ELASTICITY

The generalized theory of elasticity allows one to obtain a regular solution to problems that have a singular solution within the framework of classical elasticity [2]. To derive the corresponding equations, con- 


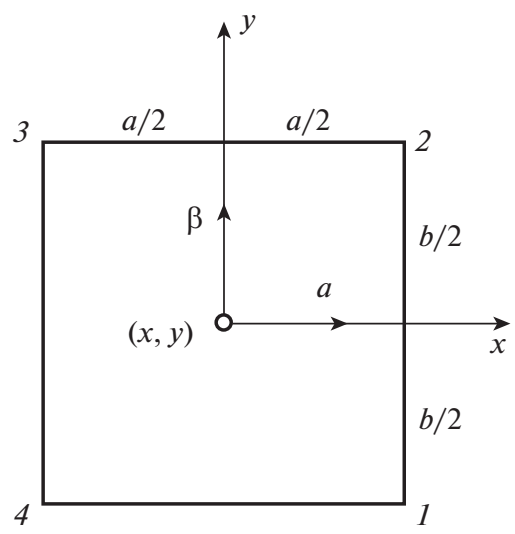

Fig. 2. Plate element.

sider the element shown in Fig. 2, which has small but finite dimensions $a$ and $b$. We introduce local coordinates $\alpha$ and $\beta$ such that $-a / 2 \leq \alpha \leq a / 2,-b / 2 \leq \beta \leq b / 2$. We represent the symmetric stress tensor $t\left(t_{x}, t_{y}, t_{x y}=t_{y x}\right)$ by the Taylor series in the vicinity of the point $(x, y)$, i.e.

$$
\begin{gathered}
t(x, y ; \alpha, \beta)=t(x, y)+\alpha \frac{\partial t}{\partial x}+\beta \frac{\partial t}{\partial y}+\frac{1}{2 !}\left(\alpha^{2} \frac{\partial^{2} t}{\partial x^{2}}+2 \alpha \beta \frac{\partial^{2} t}{\partial x \partial y}+\beta^{2} \frac{\partial^{2} t}{\partial y^{2}}\right) \\
+\frac{1}{3 !}\left(\alpha^{3} \frac{\partial^{3} t}{\partial x^{3}}+3 \alpha^{2} \beta \frac{\partial^{3} t}{\partial x^{2} \partial y}+3 \alpha \beta^{2} \frac{\partial^{3} t}{\partial x \partial y^{2}}+\beta^{3} \frac{\partial^{3} t}{\partial y^{3}}\right) .
\end{gathered}
$$

We restrict ourselves to the terms presented in equality (2.1) and find the resultant stresses acting on the faces 1-2 and 3-4 of the element shown in Fig. 2. Taking $\alpha= \pm a / 2$ and substituting expansion (2.1), we obtain

$$
R_{3-4}^{1-2}(t)=\int_{-b / 2}^{b / 2} t d \beta=b\left[t \pm \frac{a}{2} \frac{\partial t}{\partial x}+\frac{1}{8}\left(a^{2} \frac{\partial^{2} t}{\partial x^{2}}+\frac{b^{2}}{3} \frac{\partial^{2} t}{\partial y^{2}}\right) \pm \frac{a}{48}\left(a^{2} \frac{\partial^{3} t}{\partial x^{3}}+b^{2} \frac{\partial^{3} t}{\partial x \partial y^{2}}\right)\right](x, y) .
$$

Here, $t=t\left(t_{x}, t_{x y}\right)$ and the symbol $(x, y)$ mean that the resultant forces acting on the edges of 2-3 and 1-4 elements are obtained if we mutually replaced $x, y ; \alpha, \beta$ and $a, b$. The equilibrium equations of an element have the form

$$
\begin{aligned}
& R_{1-2}\left(t_{x}\right)-R_{3-4}\left(t_{x}\right)+R_{2-3}\left(t_{y x}\right)-R_{1-4}\left(t_{y x}\right)=0, \\
& R_{2-3}\left(t_{y}\right)-R_{1-4}\left(t_{y}\right)+R_{1-2}\left(t_{x y}\right)-R_{3-4}\left(t_{x y}\right)=0 .
\end{aligned}
$$

Substituting here the resultant $R$, one can obtain the differential equilibrium equations of the plane problem. Omitting further transformations described in [2] for the case $a=b$, we write the equilibrium equations in the final form

$$
\frac{\partial T_{x}}{\partial x}+\frac{\partial T_{y x}}{\partial y}=0, \quad \frac{\partial T_{y}}{\partial y}+\frac{\partial T_{x y}}{\partial x}=0
$$

where

$$
T\left(T_{x}, T_{y}, T_{x y}\right)=t-L(t), \quad L(f)=s^{2} \frac{\partial^{2} f}{\partial x^{2}}+r^{2} \frac{\partial^{2} f}{\partial y^{2}} .
$$

Here, $T$ is the generalized stress, expressed in terms of the traditional stress according to formulas (2.3), and $s$ and $r$ are structural parameters expressed in terms of the dimensions of the element $a$ and $b$ [2].

By analogy with generalized stresses, we introduce generalized deformations (Fig. 2)

$$
E_{x x}=\frac{1}{a b} \int_{-b / 2}^{b / 2}\left[u_{x}(x, y ; \alpha=a / 2, \beta)-u_{x}(x, y ; \alpha=-a / 2, \beta] d \beta, \quad E_{x y}=\gamma_{x}+\gamma_{y} \quad(x, y)\right. \text {. }
$$




$$
\gamma_{x}=\frac{1}{a b} \int_{-a / 2}^{a / 2}\left[u_{x}(x, y ; \alpha, \beta=b / 2)-u_{x}(x, y ; \alpha, \beta=-b / 2)\right] d \alpha(x, y) .
$$

Here, $u$ is the displacement of the point $(x, y)$ (Fig. 2). Suppose that the displacements can be represented in the vicinity of the point $(x, y)$ by expansions similar to equality $(2.1)$. Then the generalized deformations (2.4) take the following final form:

$$
E_{x x}=\frac{\partial U_{x}}{\partial x}, \quad E_{y y}=\frac{\partial U_{y}}{\partial y}, \quad E_{x y}=\frac{\partial U_{x}}{\partial y}+\frac{\partial U_{y}}{\partial x},
$$

where

$$
U\left(U_{x}, U_{y}\right)=u-L(u)
$$

is the generalized displacement and $L$ is the operator defined by the second equality (2.3).

For an orthotropic material, generalized stresses are related to generalized deformations as follows:

$$
T_{x}=\frac{E_{x}}{1-v_{x y} v_{y x}}\left(E_{x x}+v_{x y} E_{y y}\right) \quad(x, y), \quad T_{x y}=G_{x y} E_{x y} .
$$

For $r=s=0$, relations (2.7) degenerate into the traditional Hooke's law. Elastic constants $E, v, G$ are determined from experiments in which the stress-strain state of the material is homogeneous. In this case, the operator $L(f)=0$ and the generalized stresses and strains coincide with the traditional ones. Thus, the elasticity relations (2.7) include traditional elastic constants. In addition, the obtained relations contain two structural parameters $s$ and $r$, which are determined experimentally in relation to the problem under consideration.

Equations (2.2), (2.5), and (2.7) coincide in form with the corresponding equations of the classical theory of elasticity only, instead of the traditional stresses and displacements $t$ and $u$, they include generalized characteristics $T$ and $U$. As a solution to system (2.2), (2.5), and (2.7), one can use a solution corresponding to the classical theory of elasticity, which determines the generalized stresses and displacements $T, U$. Traditional stresses and displacements $t, u$ are found by integrating the Helmholtz equations (2.3) and (2.6). If the classical solution has no singularities and agrees with experiment, then $s=r=0$ and the generalized solution degenerates into the classical one. If the classical solution has a singularity, then the solution of the additional Helmholtz equation allows us to eliminate it.

\section{GENERALIZED SOLUTION TO THE CRACK PROBLEM}

As follows from equalities (1.6), the singularity of the solution manifests itself on the crack axis at $x=c$. In this regard, we will use a particular form of the equations of the generalized theory of elasticity obtained in the previous section, taking $b=d y$, that is, we assume that the size of the element shown in Fig. 2 is finite in the direction of the $x$ axis and infinitesimal in the direction of the $y$ axis. Then, in the relations obtained above, it is necessary to take $r=0$. As shown in [3] for an isotropic material, this approach has satisfactory accuracy in relation to the experiment and the solution of the two-dimensional problem [4]. Thus, Eq. (2.3) for stresses $t_{x}=\sigma_{x}$ and $t_{y}=\sigma_{y}$ takes the form

$$
\sigma_{x}-s^{2} \frac{\partial^{2} \sigma_{x}}{\partial x^{2}}=T_{x}, \quad \sigma_{y}-s^{2} \frac{\partial^{2} \sigma_{y}}{\partial x^{2}}=T_{y}
$$

where, in accordance with equalities (1.7) and (1.8)

$$
T_{x}=\sqrt{\frac{E_{x}}{E_{y}} \sigma_{0}}\left(\frac{x}{\sqrt{x^{2}-c^{2}}}-1\right), \quad T_{y}=\frac{\sigma_{0} x}{\sqrt{x^{2}-c^{2}}} .
$$

Consider the stress $\sigma_{y}$. This stress does not include elastic constants, so the equation

$$
\sigma_{y}-s^{2} \frac{d^{2} \sigma_{y}}{d x^{2}}=\frac{\sigma_{0} x}{\sqrt{x^{2}-c^{2}}}
$$

is similar to the corresponding equation for an isotropic plate, and its general solution has the form [3] 


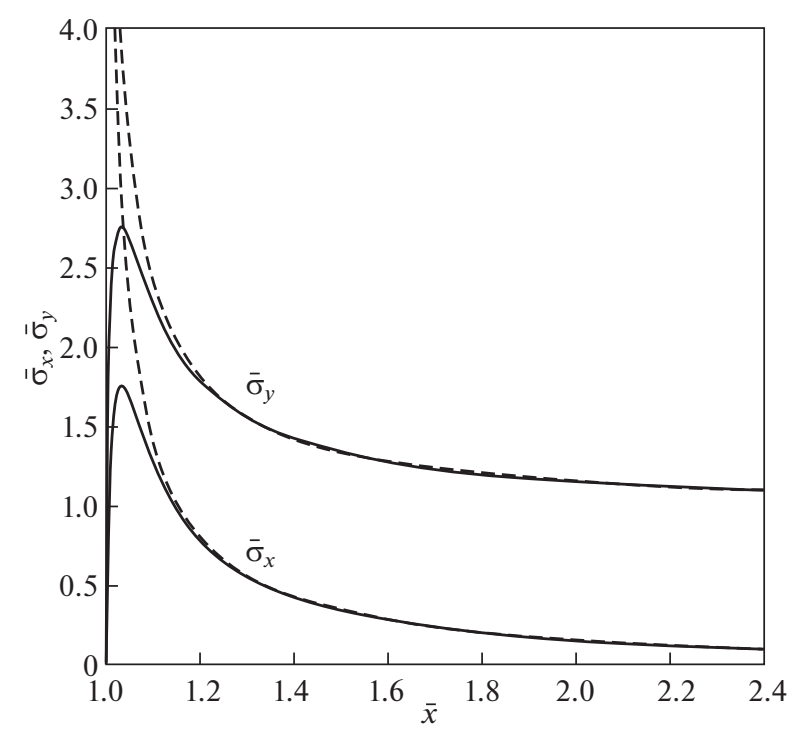

Fig. 3. Dependence of relative stresses on $\bar{x}$ at $\bar{x} \geq 1$.

$$
\bar{\sigma}_{y}(\bar{x})=C_{1} e^{-\lambda \bar{x}}+C_{2} e^{\lambda \bar{x}}+\frac{1}{2} \lambda\left(e^{-\lambda \bar{x}} \int_{1}^{\bar{x}} \frac{\bar{x} e^{\lambda \bar{x}} d \bar{x}}{\sqrt{\bar{x}^{2}-1}}-e^{\lambda \bar{x}} \int_{1}^{\bar{x}} \frac{\bar{x} e^{-\lambda \bar{x}} d \bar{x}}{\sqrt{\bar{x}^{2}-1}}\right),
$$

Here, $\bar{\sigma}=\sigma / \sigma_{0}, \lambda=c / s$ and $\bar{x}=x / c$. The regularity condition for the solution at $\bar{x} \rightarrow \infty$ is satisfied if we take

$$
C_{2}=\lambda \int_{1}^{\infty} \frac{\bar{x} e^{-\lambda \bar{x}} d \bar{x}}{\sqrt{\bar{x}^{2}-1}}
$$

Determining the constant $C_{1}$ from the condition at the end of the crack $\bar{\sigma}_{y}(\bar{x}=1)=0$, we finally obtain the following expression for the stress on the $\bar{x}$ axis at $\bar{x} \geq 1$ :

$$
\bar{\sigma}_{y}(\bar{x})=\frac{1}{2} \lambda\left(e^{-\lambda \bar{x}} \int_{1}^{\bar{x}} \frac{\bar{x} e^{\lambda \bar{x}} d \bar{x}}{\sqrt{\bar{x}^{2}-1}}+e^{\lambda \bar{x}} \int_{\bar{x}}^{\infty} \frac{\bar{x} e^{-\lambda \bar{x}} d \bar{x}}{\sqrt{\bar{x}^{2}-1}}-e^{\lambda(2-\bar{x})} \int_{1}^{\infty} \frac{\bar{x} e^{-\lambda \bar{x}} d \bar{x}}{\sqrt{\bar{x}^{2}-1}}\right) .
$$

The solution to the first equation (3.1) for $\sigma_{x}$ has the form

$$
\bar{\sigma}_{x}=\sqrt{\frac{E_{x}}{E_{y}}}\left[\bar{\sigma}_{y}(\bar{x})-\sigma_{0}\right]
$$

Solutions (3.3) and (3.4), in contrast to the classical solutions (1.7) and (1.8), are not singular. Dependences of stresses $\bar{\sigma}_{x, y}$ on the coordinate $\bar{x} \geq 1$ at $\lambda=50$ and are shown in Fig. 3 by solid lines. The dashed lines correspond to the classical solution (1.7) and (1.8).

\section{EXPERIMENTAL STUDY}

The experiment was carried out on fiberglass and carbon fiber plates, in which the reinforcement directions coincide with the $x$ and $y$ axes (Fig. 1).

Elastic constants of fiberglass are $E_{x}=23.6 \mathrm{GPa}, E_{y}=27.5 \mathrm{MPa}, G_{x y}=5.8 \mathrm{GPa}, v_{x y}=0.1, v_{y x}=$ 0.116 . Tensile strength is $s_{x}=340 \mathrm{MPa}$ and $s_{y}=416 \mathrm{MPa}$. The test plates were $250 \mathrm{~mm}$ long, $40 \mathrm{~mm}$ wide, and $1.12 \mathrm{~mm}$ thick Cracks with lengths of 5, 10, 15 and $20 \mathrm{~mm}$ were cut in the middle of the longitudinal edge of the stretched plates. It should be noted that the edge crack experiment is described here by solving the central crack problem (Fig. 1). The possibility of such an approach is based on the asymptotic analysis of the stress state near the crack tip [5], according to which this state weakly depends on the loading conditions far from the crack and the crack shape. Figure 4 shows the stress strain diagrams for plates 


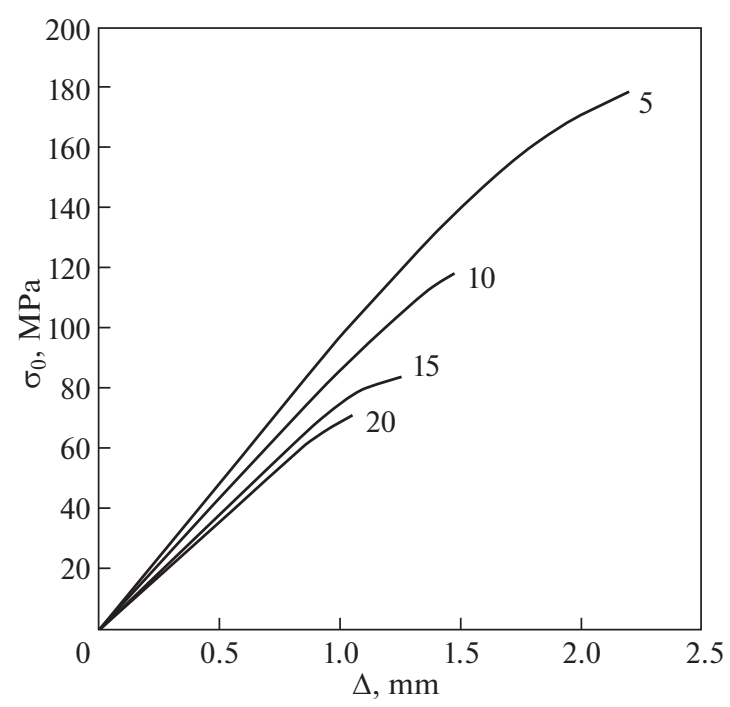

Fig. 4. Deformation diagram of plates with cracks of various lengths.

with cracks of various lengths in the direction of the $y$ axis. The numbers on the curves correspond to the crack lengths in $\mathrm{mm}$. The stress $\sigma_{0}$ is measured in $\mathrm{MPa}$, and the $\Delta$ value on the horizontal axis determines the mutual displacement in $\mathrm{mm}$ of the test machine grips on a $175 \mathrm{~mm}$ base The maximum stresses acting in the plate near the crack are represented as follows: $\sigma_{x}^{m}=k_{x} \sigma_{o}$ and $\sigma_{y}^{m}=k_{y} \sigma_{0}$, where $k_{x}$ and $k_{y}$ are the stress concentration coefficients in the vicinity of the crack. Using solution (3.3) and (3.4) for the experimental plate, it is possible to plot the dependences of $k_{x}$ and $k_{y}$ on the parameter $\lambda$, shown in Fig. 5. To assess the strength of a plate with a crack, we use the quadratic strength criterion [6]

$$
\left(\frac{\sigma_{x}^{m}}{s_{x}}\right)^{2}+\left(\frac{\sigma_{y}^{m}}{s_{y}}\right)^{2}=1,
$$

where $s_{x, y}$ is the ultimate strength of the material. The limiting curve corresponding to criterion (4.1) is in good agreement with the experiment for glass fiber laminate (Fig. 6). Expressing stresses in terms of concentration coefficients and using criterion (4.1), we can obtain the following dependence for the ultimate stress stretching the plate:

$$
\bar{\sigma}_{0}=\frac{1}{\sqrt{\left(\frac{k_{x}}{s_{x}}\right)^{2}+\left(\frac{k_{y}}{s_{y}}\right)^{2}}} .
$$

The dependence of $\bar{\sigma}_{0}$, measured in MPa, on the parameter $\lambda$, plotted using the curves shown in Fig. 5 , is shown in Fig. 7.

Determination of the breaking stress $\bar{\sigma}_{0}$ is carried out as follows. For a plate with a crack $5 \mathrm{~mm}$ long (curve 5 in Fig. 4), the experimental fracture stress is $\sigma_{0}=179 \mathrm{MPa}$. According to the graph in Fig. 7, we find the corresponding value of the parameter $\lambda=20$ and the scale factor $s=c / \lambda=0.25 \mathrm{~mm}$. The main idea of the further calculation is that the parameter $s$ is considered independent of the crack length. Then, for a plate with a crack $10 \mathrm{~mm}$ long, we obtain $\lambda=c / s=40$ and from Fig. 7 it follows that $\bar{\sigma}_{0}=114 \mathrm{MPa}$. The corresponding experimental result (curve $10 \mathrm{in} \mathrm{Fig.} \mathrm{4)} \mathrm{is} \bar{\sigma}_{0}=118 \mathrm{MPa}$. The calculation results are presented in Table 1.

As follows from Table 1, the proposed method satisfactorily predicts the fracture stress for cracked plates. It should be noted that in the last plate, for which the error reaches $10 \%$, the crack length is half the width of the plate.

The carbon fiber plates had a special hybrid structure, that is, they were formed from unidirectional carbon fiber, cross-stitched with glass threads. The elastic constants of the material required for the calculation are $E_{x}=14 \mathrm{GPa}, E_{y}=75.2 \mathrm{GPa}$. Tensile strengths are $s_{x}=170.5 \mathrm{MPa}, s_{y}=1150 \mathrm{MPa}$. The 


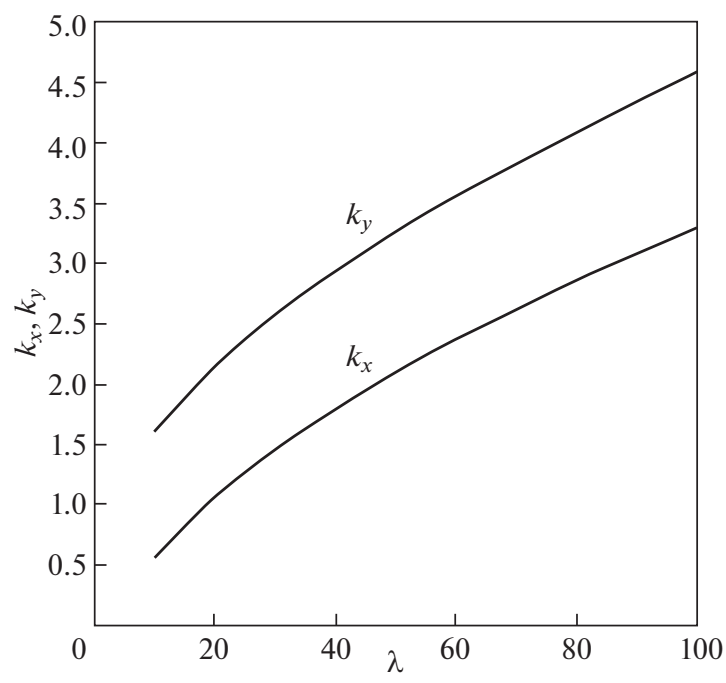

Fig. 5. Dependences of the stress concentration factors on the parameter $\lambda$.

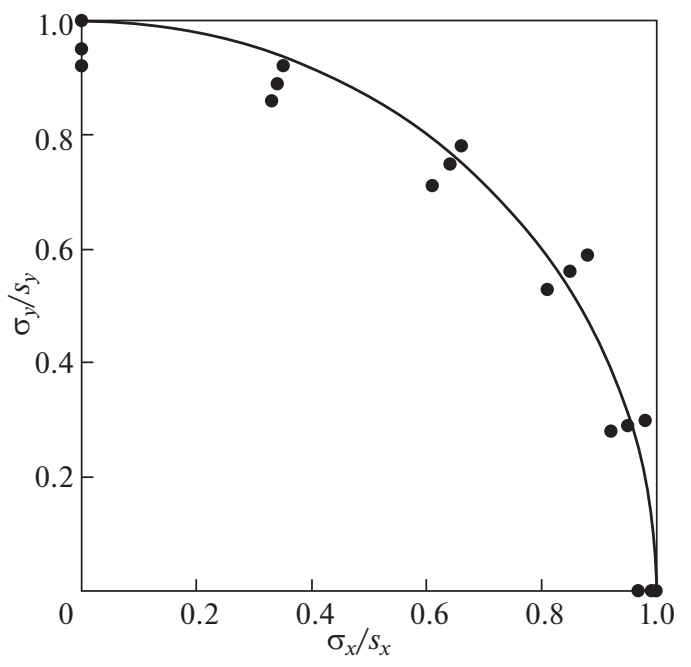

Fig. 6. Limiting curve for fibergalss fabric $(-)$ and experimental results $(\bullet)$.

samples are $30 \mathrm{~mm}$ wide and $1.5 \mathrm{~mm}$ thick. Cracks with lengths of 3, 6, 9, and $12 \mathrm{~mm}$ were applied to the longitudinal edges of the samples loaded with tension. Since the strength and stiffness of the plates under tension in the longitudinal direction (y, Fig. 1) are much higher than the corresponding characteristics for the transverse direction $(x)$, the criterion of maximum stresses can be used to assess the strength of plates made of the material under consideration. In this case, the curve for $k_{y}$ in Fig. 5 can be used to determine the stress concentration factor. The calculation is carried out by the method described above. For a plate with a crack $3 \mathrm{~mm}$ long, the limiting stress $\bar{\sigma}_{0}=690 \mathrm{MPa}$ was experimentally obtained, which corre-

Table 1. Calculated and experimental values of stresses for fiberglass plates with cracks of various lengths

\begin{tabular}{c|c|c|c|c|c}
\hline $\begin{array}{c}\text { Crack length } c, \\
\mathrm{~mm}\end{array}$ & $\begin{array}{c}\text { Parameter } s, \\
\mathrm{~mm}\end{array}$ & Parameter $\lambda$ & $\begin{array}{c}\text { Calculated } \\
\text { ultimate stress, } \\
\mathrm{MPa}\end{array}$ & $\begin{array}{c}\text { Experimental } \\
\text { ultimate stress, } \\
\mathrm{MPa}\end{array}$ & Error, \% \\
\hline 10 & 0.25 & 40 & 114 & 118 & 3.4 \\
15 & 0.25 & 60 & 90 & 84 & 7.1 \\
20 & 0.25 & 80 & 78 & 71 & 9.8 \\
\hline
\end{tabular}




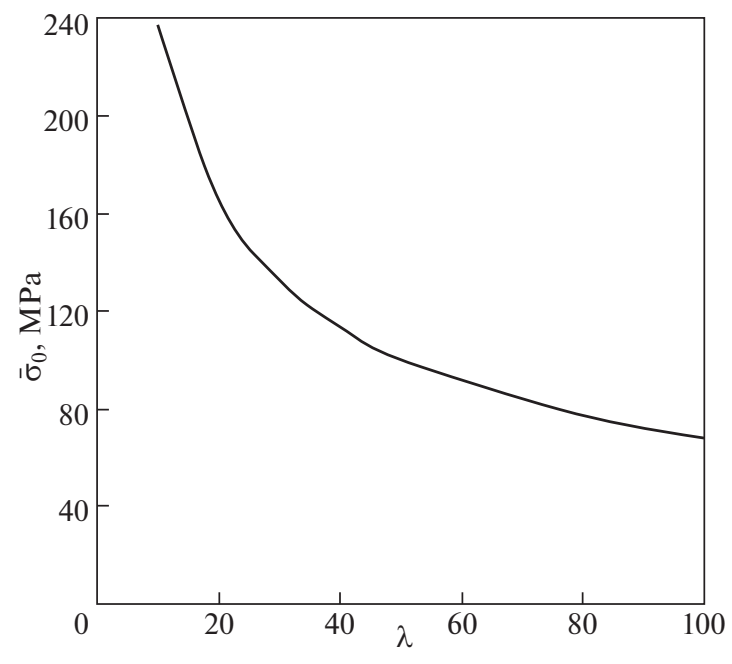

Fig. 7. Dependence of the limiting stress on the parameter $\lambda$.

Table 2. Calculated and experimental values of ultimate stress for carbon fiber plates with cracks of various lengths

\begin{tabular}{c|c|c|c|c|c}
\hline $\begin{array}{c}\text { Crack length } c, \\
\mathrm{~mm}\end{array}$ & $\begin{array}{c}\text { Parameter } s, \\
\mathrm{~mm}\end{array}$ & Parameter $\lambda$ & $\begin{array}{c}\text { Calculated } \\
\text { ultimate stress, } \\
\text { MPa }\end{array}$ & $\begin{array}{c}\text { Experimental } \\
\text { ultimate stress, }\end{array}$ & Error, \% \\
\hline 6 & 0.27 & 22.2 & 523 & 549 & 4.7 \\
9 & 0.27 & 33.3 & 432 & 441 & 2 \\
12 & 0.27 & 44.4 & 370 & 353 & 4.8 \\
\hline
\end{tabular}

sponds to the stress concentration coefficient $k_{y}=s_{y} / \bar{\sigma}_{0}=1.67$. According to the graph in Fig. 5, we find $\lambda=11$ and the parameter $s=c / \lambda=0.27$. For a plate with a crack length of $6 \mathrm{~mm}$ at the found value of the parameter $s$ we have $\lambda=c / s=22.2$, which corresponds to $k_{y}=2.2$ and the limiting stress $\bar{\sigma}_{0}=523 \mathrm{MPa}$. The corresponding experimental value is $\bar{\sigma}_{0}=549 \mathrm{MPa}$. The calculation results are presented in Table 2 .

Table 2 confirms the satisfactory accuracy of the method.

\section{CONCLUSIONS}

Thus, according to the proposed method, the problem of analysis for a plate with a crack is reduced to the traditional problem of stress concentration. For a plate with given elastic characteristics and crack length, the scale parameter $s$ is experimentally determined, which is assumed to be independent of the crack length and determines the stress concentration coefficient in the vicinity of the crack tip.

\section{FUNDING}

This work was supported by the Russian Foundation for Basic Research, grant no. 19-01-00355.

\section{OPEN ACCESS}

This article is licensed under a Creative Commons Attribution 4.0 International License, which permits use, sharing, adaptation, distribution and reproduction in any medium or format, as long as you give appropriate credit to the original author(s) and the source, provide a link to the Creative Commons license, and indicate if changes were made. The images or other third party material in this article are included in the article's Creative Commons license, unless indicated otherwise in a credit line to the material. If material is not included in the article's Creative Commons license and your intended use is not permitted by statutory regulation or exceeds the permitted use, you will need to obtain permission directly from the copyright holder. To view a copy of this license, visit http://creativecommons.org/licenses/by/4.0/. 


\section{REFERENCES}

1. Yu. N. Rabotnov, Mechanics of Deformable Solids (Nauka, Moscow, 1979) [in Russian].

2. V. V. Vasil'ev and S. A. Lurie, "Generalized theory of elasticity," Mech. Solids 50, 379-388 (2015). https://doi.org/10.3103/S0025654415040032

3. V. V. Vasil'ev and S. A. Lurie, "New solution of the plane problem for an equilibrium crack," Mech. Solids 51, $557-561$ (2016). https://doi.org/10.3103/S0025654416050071

4. V. V. Vasil'ev and S. A. Lurie, "New method for studying the strength of brittle bodies with cracks," Russ. Metall. 2020, 291-297 (2020). https://doi.org/10.1134/S0036029520040345

5. V. V. Vasil'ev, S. A. Lurie, and V. A. Salov, "Estimation of the strength of plates with cracks based on the maximum stress criterion in a scale-dependent generalized theory of elasticity," Phys. Mesomech. 22, 456-462 (2019). https://doi.org/10.1134/S102995991906002X

6. I. I. Goldenblat and V. A. Kopnov, Strength and Plasticity Criteria for Structural Materials (Mashinostroenie, Moscow, 1968) [in Russian].

Translated by I. K. Katuev 